\title{
The effect of temperature on torque motor air gap reluctance and permanent magnet polarization magnetomotive force
}

\author{
Jiayan Qin ${ }^{\mathrm{a}}$, Changchun Li, Hao Yan, Lei Li and Chan Xue \\ School of Mechanical, Electronic and Control Engineering, Beijing Jiaotong University, Beijing, China
}

\begin{abstract}
The temperaturefactor is introduced in modeling progress, based on double nozzle baffle electro-hydraulic servo valve torque motor. A new temperature model of air gap reluctance and polarization magnetomotive force was proposed. And an accurate magnetic flux computing method was deduced, in order to provide the basis for the establishment of a new torque motor model and the servo valve temperature characteristic analysis.
\end{abstract}

Keywords: torque motor; temperature; reluctance; polarization magnetomotive force.

\section{Introduction}

Rocket launcher process requires to tolerance the limit high temperature. Because of the effect of high temperature gas, oil temperature of servo hydraulic can rise up to about $150^{\circ} \mathrm{C}$ from the room time during $80 \mathrm{~s}$. The temperature of the servo valve torque motor will rise to close to the oil temperature in a very short time. Therefore, the analysis of thermal characteristics at high temperature is very necessary.

In the domestic study, Qi-xin Xiao proposed the qualitative impact of temperature for each hydraulic servo valve element comprising a torque motor as well as the oil viscosity. Chang-hai Liu conducted electromagnetic torque of motor torque and the magnetic circuit theoretical deduction by Kirchhoff's law, and established a new model. Foreign scholars Merritt E. H deduced an ideal mathematical model using air gap reluctance as a starting point[9].And the existing theoretical models of torque motor using empirical formula which is lack of temperature factor.

In this article, reluctance of permanent magnet and polarization magnetic motive force are put as the starting point, the impact of temperature on it is discussed, the original formula is corrected, and a theoretical basis for the establishment of a new model of torque motor is provided at the high temperature.

\section{Parameter calculation of torque motor}

The role of the torque motor is to convert electrical signals to mechanical movement. It's composed of permanent magnet, top yoke, bottom yoke, armature, coil, flexure tube and other components.

Figure 1a depicts a torque motor magnetic circuit schematic diagram. We can assume that reluctance of magnetic material and non-working air gap is negligible. The torque motor magnetic circuit can be represented by equivalent magnetic circuit shown in Figure 1b.

${ }^{a}$ Corresponding author : 14121251@bjtu.edu.cn

(C) 2016. The authors - Published by Atlantis Press 
When the armature is located in the median, reluctance of each working air gap is: ${ }_{R_{g}}=l_{g} / \mu_{0} A_{g}{ }^{l} l_{g}$ - the length of each of the air gap when armature is at the median position; $A_{g}$ - the area of the magnetic pole faces; $\mu_{0}$ - the permeability of air, $4 \pi \times 10^{-7} \mathrm{~Wb} / \mathrm{mA}$.
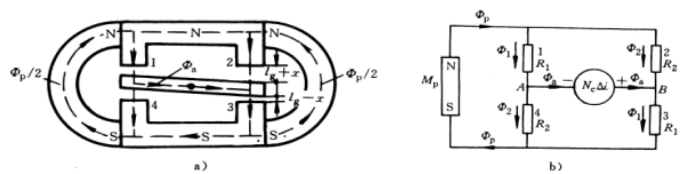

Figure 1. Torque motor magnetic circuit schematic diagram

When the armature deviates from the median position for the displacement of $\mathrm{x}$, reluctance of air gap is:

$$
R_{1}=\frac{l_{g}-x}{\mu_{0} A_{g}}=R_{g}\left(1-\frac{x}{l_{g}}\right) \quad R_{2}=\frac{l_{g}+x}{\mu_{0} A_{g}}=R_{g}\left(1+\frac{x}{l_{g}}\right)
$$

Excluding the effect of temperature on the reluctance of the air gap, there is: $R_{1}=R_{3}, R_{2}=R_{4}$, where $R_{1}$-reluctance of air gap $1 ; R_{2}$-reluctance of air gap $2 ; x$ - the displacement that the armature is deviates from the median position.

\section{Effect of temperature on the reluctance torque motors}

The permanent magnet of torque motors usually use A1-Ni-Co permanent magnet alloy. The materials that flexure tube usually use $3 \mathrm{~J} 1$ alloy. The materials that top and bottom yoke and armature usually use $1 \mathrm{~J} 50$ alloy[3].Thermal expansion of materials is usually expressed as linear expansion coefficient $\alpha$ [5].The material and expansion coefficient of each torque motor part is as follows:

Table 1. The material and expansion coefficient of each torque motor part

\begin{tabular}{lll}
\hline Part name & $\begin{array}{l}\text { Material } \\
\text { name }\end{array}$ & $\alpha / 10^{-6}\left({ }^{\circ} \mathrm{C}\right)^{-1}$ \\
\hline Permanent magnets & A1-Ni-Co & 11 \\
\hline $\begin{array}{l}\text { The top and bottom } \\
\text { yoke }\end{array}$ & $1 \mathrm{~J} 50$ & 9.2 \\
\hline armature & $1 \mathrm{~J} 50$ & 9.2 \\
\hline Flexure tube & 3J1 & 17.6 \\
\hline
\end{tabular}

When the armature of torque motors is located in the median, since thermal expansion phenomenon of permanent magnet, the top and bottom yoke, the armature, and flexure tube, the length of the air gap will change, thus affect the magnetic resistance.

At room temperature $\left(20^{\circ} \mathrm{C}\right)$ the size of the torque motor components is as shown in Fig. 2 .

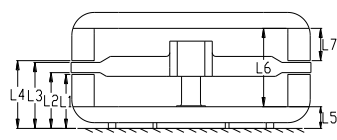

Figure 2. The size of each torque motor component

At room temperature the length of the up air gap and the down air gap is:

$$
l_{g 1}=L_{4}-L_{3}, l_{g 2}=L_{2}-L_{1}
$$

During the process that the temperature rises from room temperature to $150{ }^{\circ} \mathrm{C}$, the parts expand 
with fixation surface shown in the figure as a reference plane, The formula of the coefficient of linear expansion is ${ }^{\alpha=\frac{\Delta l}{l \cdot \Delta T}}$. There are:

$$
L_{1}^{\prime}=L_{1}\left(1+\alpha_{1} \Delta T\right), \quad L_{2}^{\prime}=L_{2}\left(1+\alpha_{2} \Delta T\right), L_{3}^{\prime}=L_{3}\left(1+\alpha_{2} \Delta T\right), L_{4}^{\prime}=L_{5}\left(1+\alpha_{1} \Delta T\right)+L_{6}\left(1+\alpha_{3} \Delta T\right)-L_{7}\left(1+\alpha_{1} \Delta T\right)
$$

Among these formulas, $\alpha_{1}$ is linear expansion coefficient of the top and bottom yoke, $\alpha_{2}$ is linear expansion coefficient of the flexure tube, $\alpha_{3}$ is linear expansion coefficient of the permanent magnet.

After heated, and in light of Eq. (3), the length of air gap is:

$$
l_{g 1}{ }^{\prime}=L_{4}^{\prime}-L_{3}^{\prime}=l_{g 1}+K_{1} \Delta T, l_{g 2}{ }^{\prime}=L_{2}^{\prime}-L_{1}^{\prime}=l_{g 2}+K_{2} \Delta T
$$

Among these equations:

$$
K_{1}=\left(L_{5}-L_{7}\right) \alpha_{1}+L_{6} \alpha_{3}-L_{3} \alpha_{2}, \quad K_{2}=L_{2} \alpha_{2}-L_{1} \alpha_{1}
$$

Put the measured actual size into the above equations, and draw curves as follows:

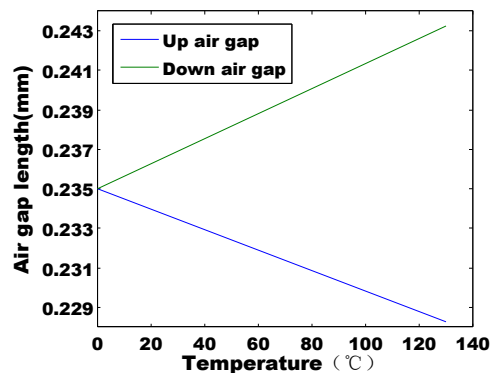

Figure 3.Thecurve that shows relation between air gap and temperature

Put Eq.(5) into Eq. (1) is:

$$
\begin{aligned}
& R_{1}=\frac{l_{g 1}+K_{1} \Delta T-x}{\mu_{0} A_{g}} R_{2}=\frac{l_{g 1}+K_{1} \Delta T+x}{\mu_{0} A_{g}} \\
& R_{3}=\frac{l_{g 2}+K_{2} \Delta T-x}{\mu_{0} A_{g}} R_{4}=\frac{l_{g 2}+K_{2} \Delta T+x}{\mu_{0} A_{g}}
\end{aligned}
$$

\section{Temperature effect on permanent magnet polarized magnetomotive force}

Magnetic material properties change as the temperature changes, When the temperature rises, the remanence $B_{r}$ and coercive force $H_{c}$ of hard magnetic material decreased, It may make changes in the operating point[4].

With the magnetic steel is magnetized, when the magnetic flux density reaches saturation magnetic flux density, it can be considered that magnetic steel has become a permanent magnet. In this case, stop magnetizing, but magnetic steel is still on the magnetizing machine, the magnetic flux density of the permanent magnet along with the B-H characteristic curve drops to ${ }_{r}$ [6][7]. 


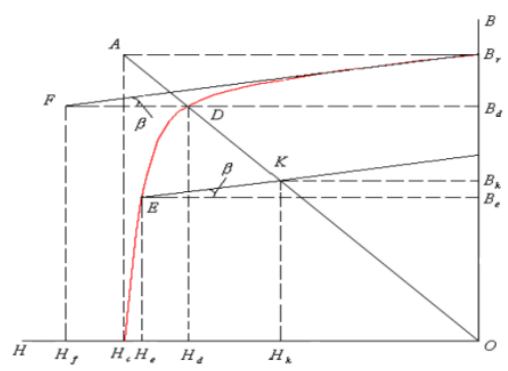

Figure 4.The B-H characteristic curve of the permanent magnet

Fig.4 depicts the B-H characteristic curve of the permanent magnet, the following formula can obtain magnetic induction intensity $B_{d}$ and magnetic field strength $H_{d}$ at the point of maximum magnetic energy product.

$$
2 B_{d} H_{c}=\rho V+B_{r} H_{c} \pm \sqrt{\left(\rho V+B_{r} H_{c}\right)^{2}-4 B_{r} H_{c} V} \quad H_{d}=\frac{V}{B_{d}}
$$

Among these formulas $\rho=2 \sqrt{\frac{B_{r} H_{c}}{V_{d}}}-\frac{B_{r} H_{c}}{V_{d}}$, is a constant related to the permanent magnet.

Point $\mathrm{E}$ is the point of maximum demagnetization(reply starting point). Although the slope of the line reply is related to the location of the starting point E, it may take approximately the tangent slope of demagnetization curve and the vertical axis intersection point $\left({ }^{F B_{r}}\right)$ to represent in engineering.

Draw parallel line at point $\mathrm{E}$, the intersection of the parallel line and the load line Is actual working point of permanent magnet.Fig.5 shows that:

$$
\mu_{k}=\tan \beta=\frac{B_{r}}{H_{c}}(1-\rho)=\frac{B_{k}-B_{e}}{H_{c}-H_{k}}
$$

In the actual operating point of the permanent magnet, there is following relationship between the magnetic flux through the permanent magnet and the magnetic induction intensity $:_{B_{k}}=\phi / A_{k}$

$\phi$ _the magnetic flux through the permanent magnet $(\mathrm{Wb})$;

$A_{k}$ - the cross sectional area of the permanent magnet $\left(\mathrm{m}^{2}\right)$;

Substituted into the Eq. (9) and arrange to obtain:

$$
\begin{gathered}
H_{k}=\phi \frac{1}{\mu_{k} A_{k}}+\left(H_{c}-\frac{B_{e}}{\mu_{k}}\right) \\
M=-H_{k} l_{k}=-\phi \frac{l_{k}}{\mu_{k} A_{k}}+l_{k}\left(-H_{c}+\frac{B_{e}}{\mu_{k}}\right)
\end{gathered}
$$

And the magnetomotive force of the permanent magnet can be expressed as:

$$
M_{0}=l_{k}\left(-H_{c}+\frac{B_{e}}{\mu_{k}}\right)=l_{k} \frac{B_{r}}{\mu_{k}}
$$

$l_{k}$ - the effective length of the permanent magnet;

$\mathrm{M}$ - magnetic motive force for external magnetic circuit provided by permanent magnet except internal magneto resistance consumption magnetomotive force of permanent magnet(A),

Put Eq. (8) into Eq. (11): 


$$
M_{0}=l_{k} \frac{H_{c}}{\left(\sqrt{\frac{B_{r} H_{c}}{V_{d}}}-1\right)^{2}}
$$

The relationship between residual magnetism density and temperature is:

$$
B_{r}=\left[1+(T-20) \alpha_{B_{r}}\right] \cdot(1-I L) \cdot B_{r 20^{\circ} \mathrm{C}}
$$

$\alpha_{B_{r}}$ - the reversible temperature coefficient of $B_{r}$;

IL-Irreversible loss rate of $B_{r}$;

$B_{r 20^{\circ} \mathrm{C}}$ - the residual magnetism density when temperature is $20^{\circ} \mathrm{C}$.

Calculate coercive force:

$$
H_{c}=\left[1+(T-20) \alpha_{B_{r}}\right] \cdot(1-I L) \cdot H_{c 20^{\circ} \mathrm{C}}
$$

$H_{c 20^{\circ} \mathrm{C}}$ - the coercive force when temperature is $20^{\circ} \mathrm{C}$.

The permanent magnet material of torque motor analyzed in this study is Al-Ni-Co permanent magnet alloys, magnetic properties of $\mathrm{Al}, \mathrm{Ni}$, and $\mathrm{Co}$ changes very little with temperature changes. The temperature coefficient of residual magnetism- $0.02 \% /^{\circ} \mathrm{C}$, irreversible loss rate is $0, B_{r 20^{\circ} \mathrm{C}}$ is $1.27 \mathrm{~T}$, $H_{c 20^{\circ} \mathrm{C}}$ is $51200 \mathrm{~A} / \mathrm{m}$, maximum magnetic energy is $52 \mathrm{KJ} / \mathrm{m}^{3}$.

Substituting the parameters into the above equations, drawn curve of relation between $\mathrm{M}$ and temperature, as figure shows:

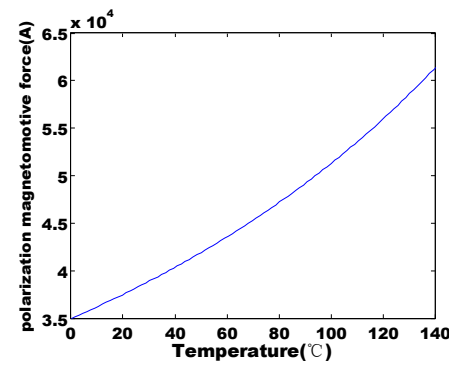

Figure 5. The curve of relation between $0 \sim 150^{\circ} \mathrm{C}$ polarization magnetomotive force and temperature

\section{Experimental results}

In order to determine the feasibility and accuracy of the theory, the experiment was carried on in this paper by using high temperature test rig.

Servo valve is fixed on the high temperature test rig. Cover torque motor with transparent shield. Install beam sensors on the top. The experiment schematic diagram is as follows:

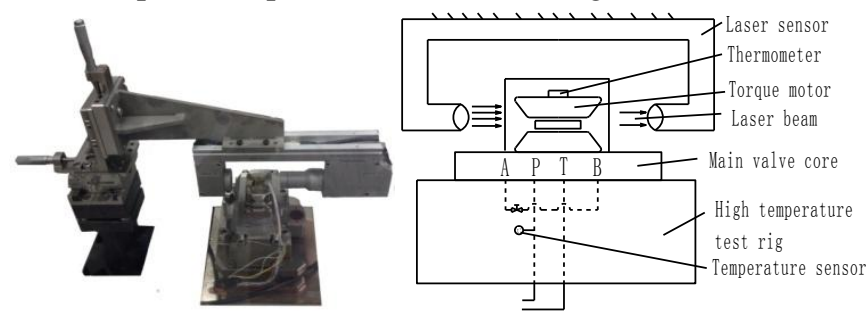

Figure 6.The experiment of temperature - air gap

It is similar to verify polarized magnetic motive force - the temperature curve. Remove the top yoke of torque motor, install a magnetometer on the position of top yoke. 

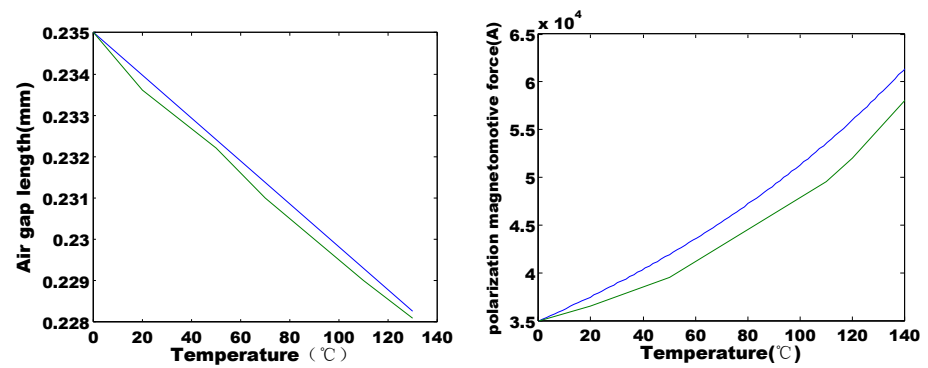

Figure 7. The curve of relation between air gap and temperature

Collate the experimental data, analyze and compare the theoretical curve and experimental curve, change strend of the two curves is consistent. How well is good.

\section{Conclusion}

It started with the structure and working principle of the torque motor, and quantitatively analysed the effect of temperature on the air gap reluctance and permanent magnet polarized magnetomotive force. It deduced linear equations and set torque motor of a certain type of servo valve as the research object. It provides the basis for the next step to carry out analysis of thermal characteristics of servo valve.[8][10]

\section{Reference}

1. YIN Yaobao. Theory and Application Technology on Electro-hydraulic Servo Control under Extreme Conditions.[M].Shanghai: Shanghai Science and Technology Press. 2011. 15-16.

2. LI Hongren. The Hydraulic Control System [M].Beijing: National Defense Industry Press.1990.

3. XIAO Qixin. Temperature Effect on Characteristics of Electro-hydraulic Servo Valve [D].Shanghai: School of Mechanical Engineering, TongjiUniversity.2009.

4. SHEN Dejun. WANGZhishan. Temperature Zero Drift of The Force Feedback Electro-hydraulic Servo Valve [J]. Chinese Hydraulics and Pneumatics. 1979.

5. YIN Yaobao[1],XIAO Qixin[1],YAN Shimin[2].Analysis on the Influence of Temperature on Electro-hydraulic Servo [J].Fluid Power Transmission and Control.2008.

6. LIU Changhai. Study on modeling and simulation of Two-stage Nozzle Flapper Servo Valve with Force Feedback [D].Harbin : Harbin Institute of Technology.2013.

7. ZHOU Anruo, MAYilong, CHEN Dengming. Study on 1J50 Soft Magnetic Alloy Temperature Stability[J].Functional Materials.2014.

8. LI Feng, MA Chengli, WANG Kai. Analysis on Electro-hydraulic Servo Valve Fault Mechanism under the Influence of Temperature [J].2014.

9. MERRITTE. H. Hydraulic Control Systems, New York: John Wieley, 1967:174-217.

10. LI Ming, WANG Wei. Calculation and Analysis of Temperature Field for BrushlessDC Torque M otor [J].MICROMOTORS. 2016. 